\title{
Tracheobronchopathia Osteochondroplastica. An underrecognized entity?
}

\author{
A. Chroneou, N. Zias, A.V. Gonzalez, J.F. Beamis Jr.
}

ABSTRACT: Tracheobronchopathia Osteochondroplastica. An underrecognized entity? A. Chroneou, N. Zias, A.V. Gonzalez, J.F. Beamis Jr.

Tracheobronchopathia osteochondroplastica (TPO) is an idiopathic disease of the trachea and major bronchi characterized by multiple submucosal osteocartilaginous nodules. The nodules may be either focal or diffuse, and typically spare the membranous wall of the airways. Symptoms are non-specific, and include dry cough, dyspnea, recurrent respiratory infections and occasionally hemoptysis. TPO is rarely considered as a diagnosis in part due to lack

\begin{abstract}
of awareness among clinicians. The diagnosis can be based on a typical bronchoscopic appearance and generally does not require biopsy of the lesions. When available, histology reveals bone formation within the submucosa with normal overlying respiratory epithelium. TPO is a benign disorder, marked by a generally favorable clinical evolution. There is currently no established treatment for the removal of airway nodules, or the prevention of further tissue growths. Interventional bronchoscopy techniques have a role in the relief of symptomatic airway obstruction, when indicated. Monaldi Arch Chest Dis 2008; 69: 2, 65-69.
\end{abstract}

Keywords: Airway diseases, Hemoptysis, Tracheobronchopathia osteochondroplastica, Chronic cough, Trachea.

Department of Pulmonary and Critical Care Medicine, Lahey Clinic Medical Center, Burlington, Massachusetts, Tufts University School of Medicine.

Correspondence: Dr. A. Chroneou, Department of Pulmonary and Critical Care Medicine, Lahey Clinic Medical Center, 41 Mall Rd., Burlington, MA 01805, U.S.A.; e-mail: chronaiou@yahoo.gr

\section{Introduction}

Tracheobronchopathia osteochondroplastica (TPO) is a rare, benign chronic disease characterized by the presence of subepithelial osteocartilaginous nodules projecting into the laryngotracheobronchial lumen. The airway projections are most frequently described as multiple lesions in the anterolateral wall of the lower two thirds of the trachea; rarely the lesions may be single and extend anywhere from the larynx to the peripheral bronchi $[1,2]$. The posterior membranous wall typically remains intact, a feature which has not been recognized in any other airway disease $[1,3]$. The nodules vary in size, and may significantly impinge on the tracheobronchial lumen $[1,3,4,5]$.

TPO was initially described by Rokitansky in 1855 [6], Luschka in 1856 [7] and Wilks in 1857 [8]. In 1947, Dalgaard reviewed 90 cases of TPO and by 1974, 245 cases had been reported worldwide [9, 10]. Mathlouthi et al. [11] counted a total of 371 reported cases of TPO in 1993. Data regarding the overall incidence of TPO vary. In autopsy studies $[12,13]$, the incidence ranges from 1:400 to $3: 1000$ while in bronchoscopy surveys $[14,15]$, the incidence ranges from $1: 125$ to $1: 6000$. TPO may appear as a rare entity in the literature because it often remains unrecognized. It may constitute an incidental finding during intubation, chest computed tomography (CT) or bronchoscopy performed for other indications [16, 17]. According to Prakash [18], TPO cases are now being more widely recognized, due to a better description of the disease in the literature and the increased awareness of bronchoscopists. Lack of awareness for this disease in the past is illustrated by the case of a patient who underwent external beam irradiation because TPO was thought to represent bronchogenic carcinoma [19]. Recently, the reported detection rate has ranged from 0.05 to $0.41 \%$ of all patients undergoing bronchoscopy [20].

\section{Etiology and pathogenesis}

The etiology of TPO is unknown. Chronic infections (e.g. mycobacteriosis), chemical or mechanical irritation (e.g. silicosis), metabolic abnormalities (e.g. amyloidosis) and genetic predisposition are proposed as causal factors [8, 21, 22]. Several reports link TPO with ozena, a disease characterized by nasal mucopurulent discharge and crusting, observed in patients with atrophic rhinitis [18, $23,24,25]$. A connection between amyloidosis and TPO has been postulated [26, 27, 28, 29]. TPO has been associated with an advanced stage of tracheobronchial amyloidosis [30]. However, in most cases of TPO, tracheobronchial amyloidosis is not present, as evidenced from reviews of large case series $[4,9]$. Congenital predisposition has also 
been suggested [6]. In this regard, few reports have described such a familial occurrence [1]. TPO has been observed with smoking but causality has never been proven $[14,31,32]$. There are also reports in the literature associating TPO with malignancies including Hodgkin's lymphoma, thyroid cancer, breast carcinoma and a case of mucoepidermoid carcinoma of the lung $[31,32,33,34]$.

The pathogenesis of this disorder also remains obscure. Various hypotheses have been proposed, originating with Virchow's viewpoint that tracheobronchial enchondromas form and subsequently develop ossification and calcium deposition [35]. The concept of airway elastic degeneration was supported by Aschoff-Freiburg who added that elastic tissue underwent metaplasia, later forming cartilage that calcified and ossified [15]. Although bone morphogenetic protein-2 and transforming growth factor $\beta$-1 are known to stimulate bone-formation [36], their role in TPO has not been elucidated [31].

\section{Clinical, laboratory and imaging features}

TPO is most commonly encountered in patients over 50 years of age. However, a case of a child with TPO has been reported [37]. No gender predominance has been described although some investigators have observed males to be affected more often than females [4, 6, 23, 38].

Clinically, there is no pathognomonic set of signs or symptoms of TPO and the majority of the patients remain asymptomatic. When TPO manifests clinically, the presentation is related to the degree of the airway obstruction and whether this occurs at the glottic, subglottic or tracheobronchial level [39]. Leske and associates, in a series of 41 patients, found that cough, hoarseness, hemoptysis, dyspnea on exertion and wheezing were the most common symptoms [40]. Several mechanisms may be involved [41], including severe airway narrowing and the absence of cilia in multiple segments of the trachea [41]. In some cases where wheezing and shortness of breath were prominent, TPO was mistaken for asthma [42]. Hemoptysis may result from ulcer formation due to the rubbing of opposing nodules [4]. Although hemoptysis often precedes the discovery of TPO, no site of bleeding is found in the majority of patients, and a cause and effect relationship has not been clearly established [4]. We recently encountered a patient who presented with hemoptysis and was found to have several soft, red endobronchial lesions which histologically were consistent with TPO [43]. This might be called "soft TPO".

No characteristic pattern of pulmonary function tests has been described in patients with TPO, and many cases are associated with preserved lung function [38, 44]. However, flow-volume loops may constitute a more sensitive indicator of disease progression [35]. With severe airflow obstruction, the flow-volume loop is characterized by a plateau in both the inspiratory and expiratory limbs of the curve $[35,45]$.
Radiologic examinations, although they may be suggestive, rarely lead to the diagnosis of TPO. Standard chest radiography is usually normal. Simple tomography, although now rarely used, is said to reveal a typical pattern of "beaded" or scalloped airways $[46,47]$. On CT scan, findings associated with TPO include thickening and irregularity of the tracheal and/or bronchial walls. The broad-based airway wall protrusions are most prominent if they are calcified. In extreme cases, marked and diffuse tracheal narrowing can be seen $[18,48,49]$.

\section{Diagnosis}

Several diseases with tracheobronchial involvement must be distinguished from TPO including tracheobronchial amyloidosis, endobronchial sarcoidosis, calcifying endobronchial tuberculosis, tracheobronchial papillomatosis, Wegener's granulomatosis, relapsing polychondritis and primary neoplasms of the airways.

The diagnostic technique most likely to establish the diagnosis of TPO is bronchoscopy. When laryngeal or upper airway involvement is present, laryngoscopy may suffice to confirm the diagnosis. Despite the name of the condition, in TPO the trachea can be spared, with only lobar or more peripheral bronchi being involved. When this occurs, atelectasis or recurrent pneumonitis may complicate the disease course $[2,50]$. Reaching such peripheral lesions with the bronchoscope to arrive at a diagnosis can be difficult. Regardless of the anatomic distribution of the disease, the endoscopic findings are quite characteristic: whitish, hard spicules that convey a cobble-stoned appearance to the airway wall [8]. These findings are characteristic of the disorder (figure 1), making tissue sampling seldom required to confirm the diagnosis of TPO.

Pathology specimens when available typically display heterotopic bone formation with abnormal cartilage proliferation and calcium deposits [4]. Adipose tissue fragments, along with foci of bone marrow with active hematopoiesis, can also be present [51] (figure 2). The technical aspects of obtaining adequate tissue samples warrant mentioning. Biopsies via the flexible bronchoscope may be challenging given the nature of the lesions and the limitations of working through a small channel [9]. Larger biopsy forceps, used through a rigid bronchoscope, are more likely to yield adequate specimens. However, with significant airway narrowing, passage of the rigid bronchoscope may be difficult. A grating sensation has occasionally been described when the rigid bronchoscope is passed over the airway lesions [18].

\section{Treatment}

While TPO is generally considered a benign airway disorder, with minimal progression of the lesions [40], severe tracheal stenosis resulting from this disease process has been reported [52, 53]. Asymptomatic patients could be simply followed, even for prolonged periods of time. A follow-up of 


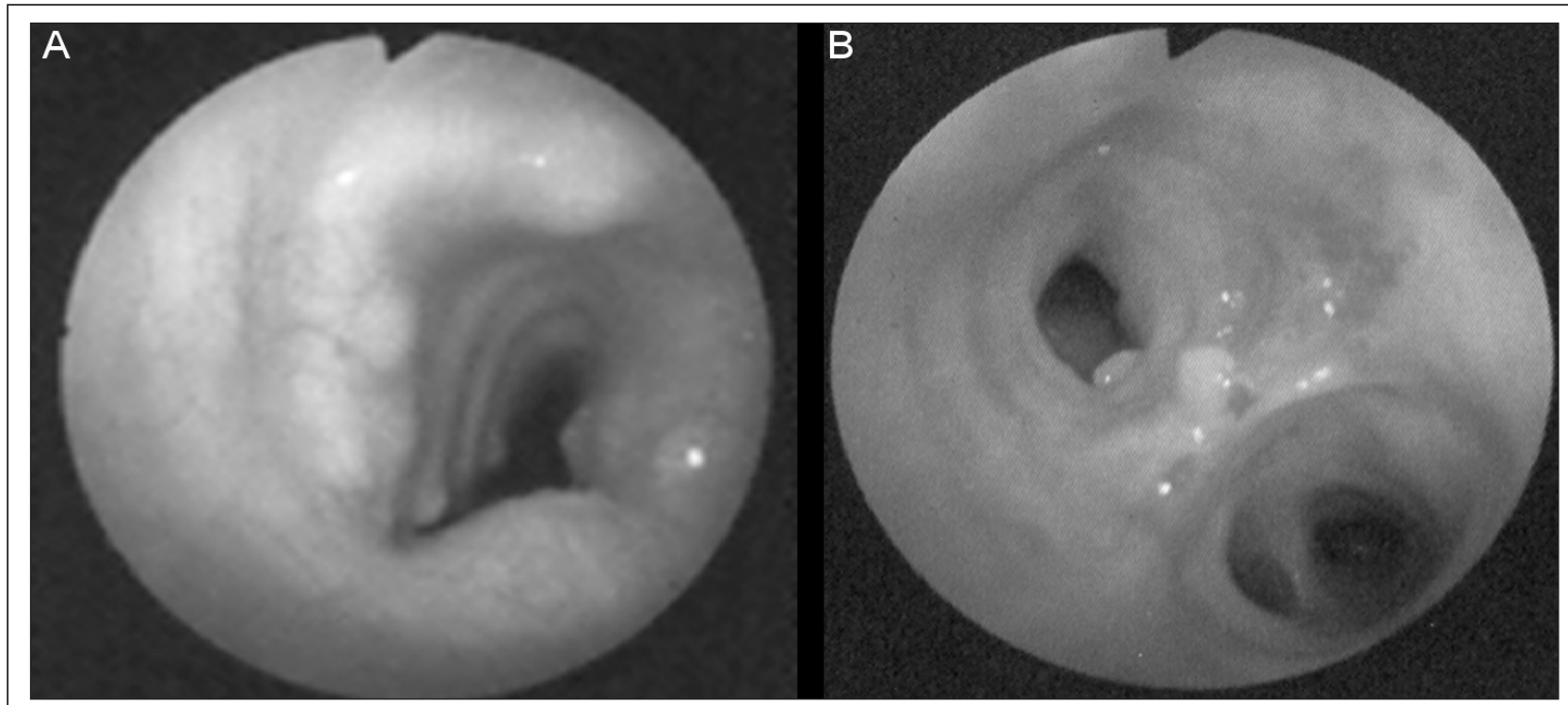

Fig. 1. - Bronchoscopic appearance of tracheobronchopathia osteochondroplastica. A. Note, the osseocartilaginous nodules are located in the anterolateral tracheal wall. The posterior membranous wall is spared. B. Left upper lobe involvement. (Courtesy of Dr Udaya B.S. Prakash, Pulmonary and Critical Care Medicine, Mayo Medical Center, Mayo Medical School, Rochester, Minnesota).

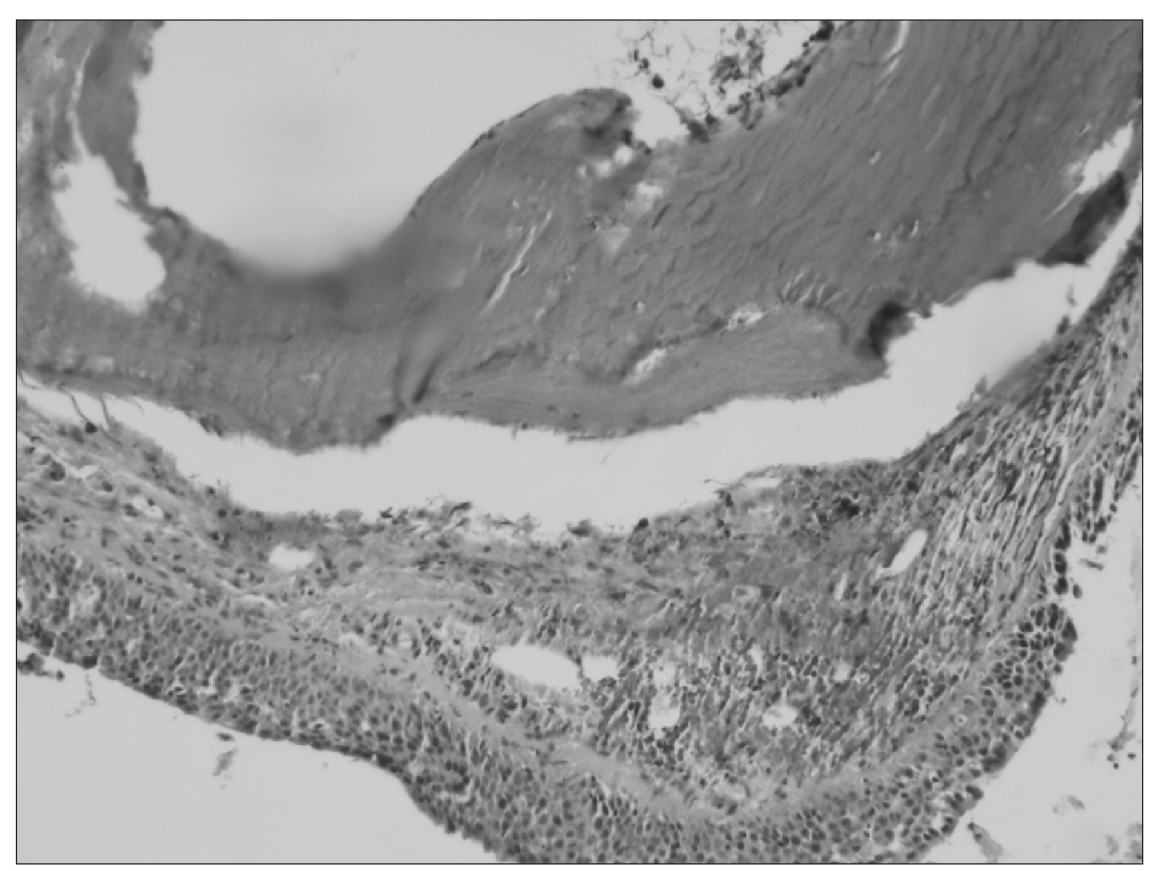

Fig. 2. - Biopsy demonstrating areas of normal epithelium along with osseous tissue with bronchial cartilage and bone marrow elements. These findings are consistent with tracheobronchopathia osteochondroplastica. Hematoxylin-Eosin, 400x.

up to 25 years has been described in the literature [54]. However, when the trachea or the bronchi are narrowed, surgical treatment (tracheal or laryngeal resection) and bronchoscopic therapy (removal of nodules with biopsy forceps, laser ablation and cryotherapy) have been attempted $[4,25,30,55$, 56, 57]. According to Prakash et al. [1] the success of endoscopic nodule removal with laser and biopsy instruments is variable, but generally results in improvement of the patient's respiratory status. External beam radiation has also been described in a very limited number of patients in the past, with reported relief of symptoms [4, 18, 46, 58].

The prognosis of TPO is usually good, though it frequently occurs in association with malignant tumors [59]. Yokoyama reported an association with lung cancer in $11.1 \%$ of their patients with TPO, with adenocarcinoma being the most common histologic subtype [59]. For patients manifesting some of the symptoms described above, conservative therapy with maintenance of airway humidity, prompt therapy of intercurrent infections and avoidance of airway irritation, are the goals that should be achieved [18].

In summary, TPO remains a rare entity, which is frequently found unexpectedly and diagnosed incidentally. It affects only the cartilaginous part of the airways, with submucosal nodules projecting into the tracheobronchial lumen. The importance of adequate awareness and recognition is underlined in this review, with the hope of preventing unnecessary diagnostic procedures. Most patients are asymptomatic or present non-specific signs and symptoms. Bronchoscopy is usually sufficient to make the diagnosis, and tissue biopsies are seldom required. No specific treatment or preventive measure seems to be necessary given the generally benign nature of this entity. However, interventional bronchoscopy techniques may be helpful in symptomatic cases even if the experience is limited due to the rarity of the disorder.

Acknowledgements: We would like to thank Dr. Udaya B.S. Prakash for providing the pictures shown in figure 1 . 


\section{References}

1. Prakash UB, McCullough AE, Edell ES, et al. Tracheopathia osteoplastica: familial occurrence. Mayo Clin Proc 1989; 64: 1091-1096.

2. Shigematsu Y, Sugio K, Yasuda M, et al. Tracheobronchopathia osteochondroplastica occurring in a subsegmental bronchus and causing obstructive pneumonia. Ann Thorac Surg 2005; 80: 1936-1938.

3. Young RH, Sandstrom RE, Mark GJ. Tracheopathia osteoplastica: clinical, radiologic, and pathological correlations. J Thorac Cardiovasc Surg 1980; 79: 537-541.

4. Nienhuis DM, Prakash UB, Edell ES. Tracheobronchopathia osteochondroplastica. Ann Otol Rhinol Laryngol 1990; 99: 689-694.

5. Wong JS, Ng CS, Yim AP. Hemoptysis with multiple tracheal nodules. Chest 2005; 128: 3671-3673.

6. Dalgaard JB. Tracheopathia chondro-osteoplastica: a case elucinating the problems concerning development and ossification of elastic cartilage. Acta Pathol Microbiol Scand 1947; 24: 118-134.

7. Moersch HJ, Broders AC, Havens FZ. Tracheopathia osteoplastica (osteoma of the trachea). Arch Otolaryngol 1937; 26: 291-293.

8. Wilks S. Ossific deposits in the larynx, trachea and bronchi. Trans Pathol Soc Lond 1857; 8: 88.

9. Martin CJ. Tracheobronchopathia osteochondroplastica. Arch Otolaryngol 1974; 100: 290-293.

10. Dalgaard JB. Tracheopathia chondro-osteoplastica. Acta Pathol Microbiol Scand 1947; 24: 118-134.

11. Mathlouthi A, Ben Rehouma C, Ben M'Rad S, et al. [Tracheobronchopathia osteochondroplastica. Personal observation and review of the literature]. Rev Pneumol Clin 1993; 49: 156-162.

12. Pedersen MF, Worsoe-Petersen J. Tracheopathia osteoplastica. J Laryngol Otol 1969; 83: 883-888.

13. Pounder DJ, Pieterse AS. Tracheopathia osteoplastica: report of four cases. Pathology 1982; 14: 429-433.

14. Smith DC, Pillai R, Gillbe CE. Tracheopathia osteochondroplastica. A cause of unexpected difficulty in tracheal intubation. Anaesthesia 1987; 42: 536-538.

15. Primer G. Trachobronchopathia osteochondroplastica. Prax Klin Pneumol 1979; 33: 1060-1063.

16. Coetmeur D, Bovyn G, Leroux P, et al. Tracheobronchopathia osteochondroplastica presenting at the time of a difficult intubation. Respir Med 1997; 91: 496-498.

17. Neumann A, Kasper D, Schultz-Coulon HJ. [Clinical aspects of tracheopathia osteoplastica]. Hno 2001; 49: 41-47.

18. Prakash UB. Tracheobronchopathia osteochondroplastica. Semin Respir Crit Care Med 2002; 23: 167-175.

19. Clerf LH. Tracheopathia osteoplastica (osteoma of the trachea). Report of a case. Ann Otol Rhinol Laryngol 1944; 53: 839-843.

20. Fujimoto K, Kumabe T, Fujitoh H. Two cases of tracheobronchopathia osteochondroplasticaand a review Of 86 cases in the Japanese literature. J Jpn Soc Bronchology 1988; 13: 650-658.

21. Baugnee PE, Delaunois LM. Mycobacterium avium-intracellulare associated with tracheobronchopathia osteochondroplastica. Eur Respir J 1995; 8: 180-182.

22. Shih JY, Hsueh PR, Chang YL, et al. Tracheal botryomycosis in a patient with tracheopathia osteochondroplastica. Thorax 1998; 53: 73-75.

23. Harma RA, Suurkari S. Tracheopathia chondro-osteoplastica. A clinical study of thirty cases. Acta Otolaryngol 1977; 84: 118-123.

24. Magro P, Garand G, Cattier B, et al. [Association of tracheobronchopathia osteochondroplastica and ozene]. Rev Mal Respir 2007; 24: 883-887.

25. Vaheri E, Vaheri E. Tracheopathia osteoplastica. Acta Otolaryngol 1967; 64: 251-255.
26. Sakula A. Tracheobronchopathia osteoplastica: its relationship to primary tracheobronchial amyloidosis. Tho$\operatorname{rax} 1$ 1968; 23: 105-110.

27. Toyoda M, Ebihara Y, Kato H, et al. Tracheobronchial AL amyloidosis: histologic, immunohistochemical, ultrastructural, and immunoelectron microscopic observations. Hum Pathol 1993; 24: 970-976.

28. Case records of the Massachusetts General Hospital. Weekly clinicopathological exercises. Case 32-1999. A 44-year-old man with tracheal narrowing and respiratory stridor. N Engl J Med 1999; 341: 1292-1299.

29. Weiss L. Isolated multiple nodular pulmonary amyloidosis. Am J Clin Pathol 1960; 33: 318-329.

30. Alroy GG, Lichtig C, Kaftori JK. Tracheobronchopathia osteoplastica: end stage of primary lung amyloidosis? Chest 1972; 61: 465-468.

31. Bioque J. Tracheobronchopathia osteochondroplastica: clinical study and follow-up in nine cases. $J$ Bronch. 2001; 8: 78-83.

32. Morita S, Yokoyama N, Yamashita S, et al. Tracheopathia osteochondroplastica complicated with thyroid cancer: case report and review of the literature in Japan. Jpn J Med 1990; 29: 637-641.

33. de Wall N, Roggenbuck C, Endres P, et al. [Tracheobronchopathia osteochondroplastica and coexistent mucoepidermoid carcinoma of the lung: Case report]. Pneumologie 1992; 46: 74-77.

34. Karlikaya C, Yuksel M, Kilicli S, et al. Tracheobronchopathia osteochondroplastica. Respirology 2000; 5: 377-380.

35. Bergeron D, Cormier Y, Desmeules M. Tracheobronchopathia osteochondroplastica. Am Rev Respir Dis 1976; 114: 803-806.

36. Tajima K, Yamakawa M, Katagiri T, et al. Immunohistochemical detection of bone morphogenetic protein-2 and transforming growth factor beta-1 in tracheopathia osteochondroplastica. Virchows Arch 1997; 431: 359-363.

37. Simsek PO, Ozcelik U, Demirkazik F, et al. Tracheobronchopathia osteochondroplastica in a 9-year-old girl. Pediatr Pulmonol 2006; 41: 95-97.

38. Lundgren R, Stjernberg NL. Tracheobronchopathia osteochondroplastica. A clinical bronchoscopic and spirometric study. Chest 1981; 80: 706-709.

39. Castella J, Puzo C, Cornudella R, et al. Tracheobronchopathia osteochondroplastica. Respiration 1981; 42: 129-134.

40. Leske V, Lazor R, Coetmeur D, et al. Tracheobronchopathia osteochondroplastica: a study of 41 patients. Medicine (Baltimore) 2001; 80: 378-390.

41. Chen AY, Donovan DT. Impaired ciliary clearance from tracheopathia osteoplastica of the upper respiratory tract. Otolaryngol Head Neck Surg 1997; 117: S102-104.

42. Hayes D, Jr. Tracheopathia osteoplastica misdiagnosed as asthma. $J$ Asthma 2007; 44: 253-255.

43. Riker Dr, Campagna Ac, Beamis Jf. Tracheobronchopathia Presenting as Hemoptysis Associated With Vascular Endobronchial Tumors. J Bronch. 2007; 14: 212-214.

44. Tukiainen H, Torkko M, Terho EO. Lung function in patients with tracheobronchopathia osteochondroplastica. Eur Respir J 1988; 1: 632-635.

45. Meyer CN, Dossing M, Broholm H. Tracheobronchopathia osteochondroplastica. Respir Med 1997; 91: 499-502.

46. Howland WJ, Jr., Good CA. The radiographic features of tracheopathia osteoplastica. Radiology 1958; 71: 847-850.

47. Whitehouse G. Tracheopathia osteoplastica. Br J Radiol 1968; 41: 701-703.

48. Onitsuka H, Hirose N, Watanabe K, et al. Computed tomography of tracheopathia osteoplastica. Am J Roentgenol 1983; 140: 268-270. 
49. Hirsch M, Tovi F, Goldstein J, et al. Diagnosis of tracheopathia osteoplastica by computed tomography. Ann Otol Rhinol Laryngol 1985; 94: 217-219.

50. Hodges MK, Israel E. Tracheobronchopathia osteochondroplastica presenting as right middle lobe collapse. Diagnosis by bronchoscopy and computerized tomography. Chest 1988; 94: 842-844.

51. Akyol MU, Martin AA, Dhurandhar N, et al. Tracheobronchopathia osteochondroplastica: a case report and a review of the literature. Ear Nose Throat $J$ 1993; 72: 347-350.

52. Case records of the Massachusetts General Hospital. Weekly clinicopathological exercises. Case 46-1992. A 48-year-old woman with a narrowed trachea. $N$ Engl $J$ Med 1992; 327: 1512-1518.

53. Molloy AR, McMahon JN. Rapid progression of tracheal stenosis associated with tracheopathia osteochondroplastica. Intensive Care Med 1988; 15: 60-62.

54. van Nierop MA, Wagenaar SS, van den Bosch JM, et al. Tracheobronchopathia osteochondroplastica. Report of four cases. Eur J Respir Dis 1983; 64: 129-133.

55. Birzgalis AR, Farrington WT, O'Keefe L, et al. Localized tracheopathia osteoplastica of the subglottis. $J$ Laryngol Otol 1993; 107: 352-353.

56. Gleich LL, Rebeiz EE, Pankratov MM, et al. The holmium: YAG laser-assisted otolaryngologic procedures. Arch Otolaryngol Head Neck Surg 1995; 121: 1162-1166.

57. O'Reilly RR, Marty AT. Tracheopathia osteoplastica: case report. Mil Med 1978; 143: 497-498.

58. Vilkman S, Keistinen T. Tracheobronchopathia osteochondroplastica. Report of a young man with severe disease and retrospective review of 18 cases. Respiration 1995; 62: 151-154.

59. Yokoyama T, Ninomiya H, Matsunami M. A case of tracheobronchopathia osteochondroplastica accompanied by lung cancer and a review of similar cases in the japanese literature. J Jpn Soc Bronchology 1996; 18: 558-562.

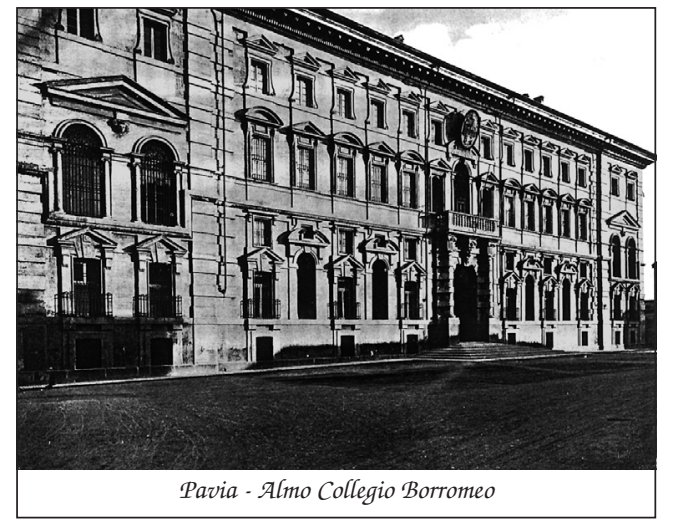

